\title{
Predictive control based upon state space models
}

\author{
JENS G. BALCHEN, DAG LJUNGQUIST AND STIG STRAND $\dagger$
}

Keywords: Optimal control, predictive control, non-linear control system, statespace models, iterative methods, on-line operation

Repetitive online computation of the control vector by solving the optimal control problem of a non-linear multivariable process with arbitrary performance indices is investigated. Two different methods are considered in the search for an optimal, parameterized control vector: Pontryagin's Maximum Principle and optimization by using the performance index and its gradient directly. Unfortunately, solving this optimization problem has turned out to be a rather time-consuming task which has resulted in a time delay that cannot be accepted when the actual process is exposed to rapidly-varying disturbances. However, an instantaneous feedback strategy operating in parallel with the original control algorithm was found to be able to cope with this problem.

\section{Introduction}

Many industrial processes which are multivariable in nature, are subject to large time-varying disturbances and have constraints imposed upon both the control and state variables. Predictive control techniques have been proposed as an online system which permits the flexibility required to handle such difficulties.

Most predictive control techniques proposed so far, are based upon nonparametric models such as an impulse response matrix or a discrete convolution matrix (Cutler and Ramaker 1980, Richalet et al. 1978). As long as the process can be described by a set of linear, differential equations, this modeling approach is applicable. In many industrial applications, however, the processes are run with a wide range of operating conditions so that non-linearities will usually occur. Internal state variables that are not defined as inputs or outputs in a linear model, may be constrained to stay within certain limits. The existing predictive control techniques are not suitable for handling such constraints in a direct and logical manner. In addition, a discrete convolution model is a 'non-minimal realization' with the result that the control algorithm derived may be wasteful in terms of computational time. The CARIMA plant model used in the generalized predictive control algorithm (Clarke et al. 1987) is another input-output description, which has some of the same faults.

In a state space description, non-linearities can easily be represented and constraints on any variable taken care of by inequalities. The state variables usually represent physical quantities and the predicted variations of the different state variables can give important information about the future. The state space description

Received November 1988.

$\uparrow$ Division of Engineering Cybernetics, The Norwegian Institute of Technology, Trondheim, Norway.

This article was presented at the 1988 American Control Conference, Atlanta, Georgia, USA, June 15-17 and is reprinted with the permission of the American Automatic Control 
constitutes a parametric model. In formulating such a model, basic physical laws representing material and energy balances and elementary information about the physical dimensions of equipment will help to establish the mathematical structure of the model. Continuous online estimation of model parameters (model identification) will improve the model to give a satisfactory representation of the process dynamics.

Reid (1981) makes use of a state space model in his approach to predictive control. Even so, this formulation is limited to quadratic performance indices, weighting the deviations in predicted output from the desired ones and sometimes weighting future changes in input. In this way a reference trajectory has to be formulated. Using arbitrary performance indices, however, optimal trajectories are always ensured although computational requirements are increased.

This paper is organized as follows; in $\S 2$ we discuss a proposal for a complete system for predictive control based upon state space models. In $\S 3$ we formulate the optimization problem, in $\S 4$ we discuss control strategies and computer requirements, while some simulation results are presented in $\S 5$.

\section{Complete system for predictive control}

An industrial program package for multivariable, predictive process control based on state space models should consist of the components shown in Figure 1 (Balchen and Mumme 1987). From an initial study of the process, we obtain the basic concept for a suitable model structure and parameter values, which have to be updated online by a structure identification and parameter estimation algorithm. Moreover a state estimator should be included, such as an extended Kalman filter. In parallel with this real time model, there is an equivalent model which operates

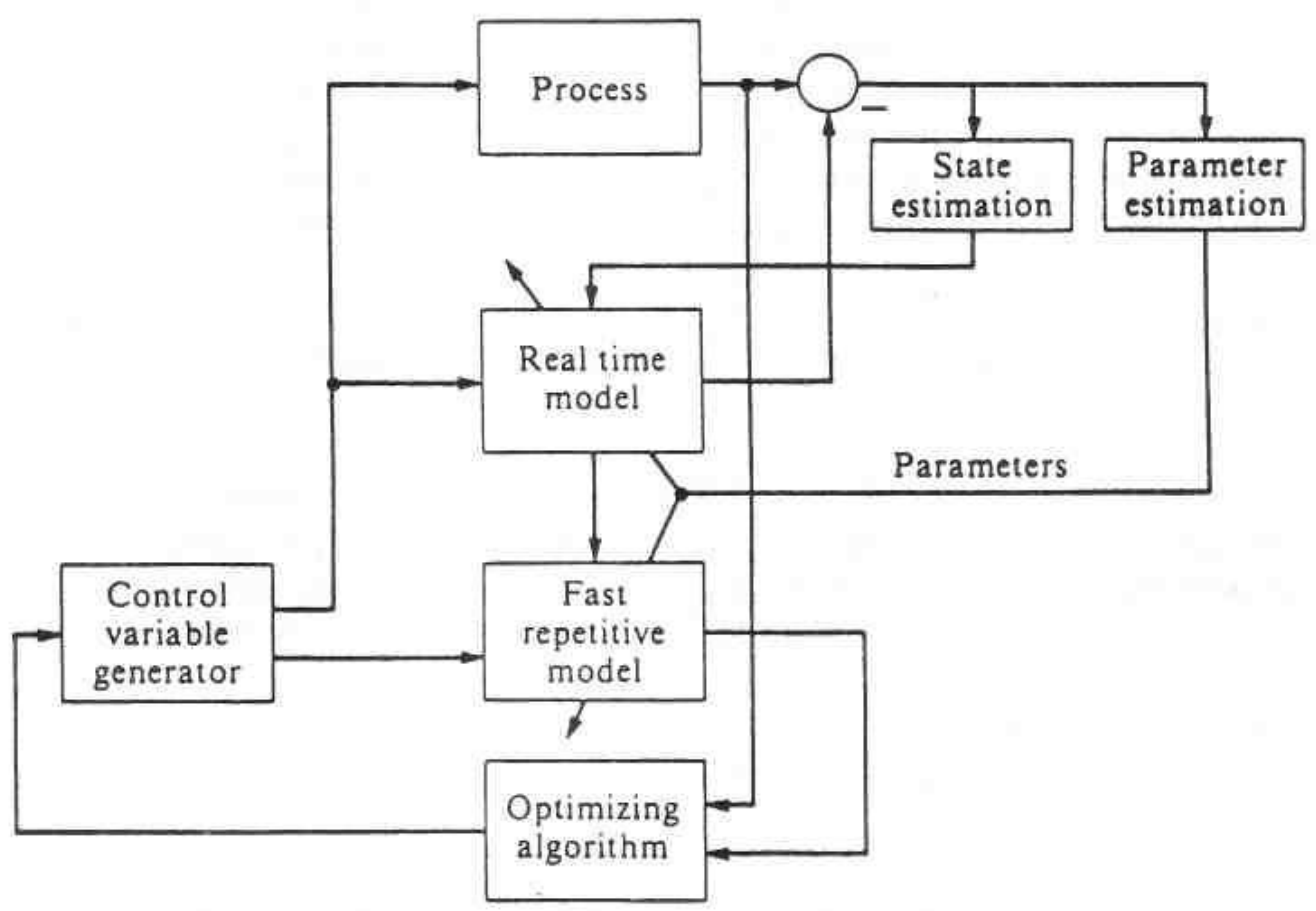

Figure 1. Components of the total system for predictive control. 
repetitively with a highly reduced time scale. The estimated states and parameters of the real time model determine the equivalent quantities in the repetitive model. This makes it possible for an iterative optimization algorithm to find a set of control variables which ensure optimality in the future. The set of control variables is recomputed repeatedly in order to take care of time-varying disturbances and changes in performance criteria. The required repetition rate for the computation of new control variables depends on the rate of change in the disturbances. In order to avoid serious problems in the future, such as saturation in the control variables or state variables exceeding constraints, each optimization has to be based on a prediction over a time horizon longer than the dominating time constants in the closed loop.

To achieve satisfactory predictions, it is important to be able to estimate and predict the most dominating disturbances. If we know the system which generates the disturbances, they can be modeled and included in the process model in Fig. 1. A slowly varying disturbance can be modeled as a Wiener process, continuously being updated by the integration of the innovation process of a Kalman filter. Nonestimated disturbances may result in steady state prediction errors. In the predictive control scheme MAC (Mehra and Mahmood 1985), correction terms are introduced to overcome this problem. This corresponds to the strategy of integrating the innovation process. The main difference is that the Kalman filter ideally generates optimal estimates of the disturbances.

In Fig. 1 we notice that the estimation of the state variables, disturbances and model parameters takes place in parallel with the computation of the optimal control variables. The optimization part therefore continuously generates a new set of control variables.

\section{Formulation of the optimization problem}

It can be seen in Fig. 1 that the development of the details in each component of the total system for predictive control can be made nearly independent of each other. In this paper we assume that we have an ideal model of the process and that the state variables are available from this model.

The problem is formulated as follows. The process is described by

$$
\dot{x}=f(x, u, v)
$$

where

$$
\begin{array}{ll}
\boldsymbol{x} & \text { state vector } \\
\boldsymbol{u} & \text { control vector } \\
\boldsymbol{v} & \text { disturbance vector } \\
\boldsymbol{f} & \text { vector of non-linear functions }
\end{array}
$$

A specification of the performance of the control system can be represented mathematically by a performance index

$$
J=S\left(x\left(t_{1}\right)\right)+\int_{t_{0}}^{t_{1}} L(x, u, t) d t
$$


where

$$
\begin{array}{ll}
t_{0} & \text { initial time for the optimization } \\
t_{1}=T+t_{0} & \text { final time for the optimization where } T \text { is the time-horizon } \\
S\left(x\left(t_{1}\right)\right) & \text { is a scalar function weighting the final value of the state vector } \\
L(x, u, t) & \begin{array}{l}
\text { is a scalar function expressing costs and profits resulting from the } \\
\text { process. }
\end{array}
\end{array}
$$

The desired control vector is now the one that minimizes (or maximizes) the performance index (2). There is no particular restriction that the function $L$ has to be a quadratic function for instance as is the case when an analytical solution is desired. An exact specification of the final state is avoided by choosing an appropriate $S$, and since the initial time state vector and the length of the time horizon are both given, this is of vital importance when an optimization algorithm is to be deduced.

Constraints on the state variables or control variables may often be expressed through relations such as

$$
C_{i}(u, t) \leqslant 0 \quad i=1, \ldots, r
$$

and

$$
Z_{j}(x, t) \leqslant 0 \quad j=1, \ldots, n
$$

How such constraints are to be handled in the minimization algorithm depends on whether they are hard or soft, i.e. whether dynamic violations of the constraints are allowed or not. Constraints on the control variables do not usually cause any problem, although the convergence of the solution will tend to be worse. As far as constraints on the state variables are concerned, difficulties may arise. So far we have experienced that penalty functions in the performance index is the best way to handle such constraints.

\section{Control strategies and computer requirements}

Solving the optimization problem appears to be a rather time-consuming task. Therefore it is of vital importance to choose the most effective algorithm and a strategy of control vector computation that ensures as optimal a solution as possible. We denote the time required to compute an optimal control vector by $\delta t$. Assuming that we want the computer to be occupied all the time, immediately after a new optimal control vector is available, we have to update the model and continue with the computation of the 'next' control vector. Updating the model during the optimization period, usually makes the convergency worse and is not to be recommended. The result is that fast changes in the disturbances or the performance criteria cannot be taken into consideration until the next optimization period. This creates a time delay of nearly two times $\delta t$ in the worst case. If it is impossible to predict the disturbances rather accurately, a strategy of control vector computation that ensures some kind of instantaneous feedback of the registered deviation from the predicted state-space variables should be used. In other words, we should find an approximation to the ideal optimal control vector that includes a feedback strategy. 
Consider the optimal control vector given by

$$
\boldsymbol{u}=\boldsymbol{g}(\boldsymbol{x}, \boldsymbol{\theta}, t)
$$

Our prediction of the future states is denoted by $\boldsymbol{x}$, while in fact we will have deviation $\delta \boldsymbol{x}$ from this prediction. A first order approximation of (5) is then given by

$$
\begin{aligned}
\boldsymbol{u} & \approx \boldsymbol{g}\left(\boldsymbol{x}^{0}, \boldsymbol{\theta}, t\right)+\left.\frac{\partial \boldsymbol{g}()}{\partial \boldsymbol{x}}\right|_{\boldsymbol{x}=\mathbf{x}^{0}} \delta \boldsymbol{x} \\
& =\boldsymbol{u}^{0}+G\left(\boldsymbol{x}-\boldsymbol{x}^{0}\right)
\end{aligned}
$$

An optimal control variable $\boldsymbol{u}^{0}$ corresponds to an optimal predicted trajectory $\boldsymbol{x}$, while $G$ is the feedback matrix.

Substitution of (6) into the state space model and performance index in order to determine $\boldsymbol{u}$ and $G$ leads to an overdetermined system of parameters in the optimization algorithm. Simulation results so far indicate that $\boldsymbol{u}^{0}$ should be chosen as the optimal, nominal control vector computed by optimizing (2), while Eqn. (1) is regarded as a constraint. A suitable $G$ matrix may be found by a specification of the eigenvalues of the closed loop system (linearized system). The constant elements of the $G$ matrix may vary as the nominal trajectory runs through the non-linear state space. $G$ then ensures an instantaneous feedback strategy which takes care of rapidly-varying disturbances, while $\boldsymbol{u}^{0}$ controls the nominal trajectory $\boldsymbol{x}^{0}$, when the predicted disturbances and changes in the operating conditions are taken into consideration.

Optimization of (2) and (1) with respect to $\boldsymbol{u}^{0}$ may be done by using Pontryagin's Maximum Principle. Generally this leads to a two point boundary value problem (TPBVP) which cannot be solved analytically. Therefore an iterative numerical solution method is required. An alternative strategy to using the Maximum Principle will be to optimize the performance index directly by using a gradient technique.

Most effort so far has been directed to the development of an effective optimization algorithm. Both methods mentioned above have been investigated and applied. In order to save critical time-consumption, a parameterized control vector has been used. Exponentially decaying weight upon future disturbances is the inherent result of traditional optimal control (LQ). According to this, an exponential distribution of the subintervals referring to the different parameters of the control vector seems to be logical. Simulation results indicate that a more moderate increase should be used.

A rough estimate of the computer costs is interesting to get an idea of the applicability of the predictive control algorithm. We assume that $3 r$ parameters are to be computed, where $r$ is the dimension of the control vector (i.e. each control variable is represented by three parameters). We also assume that some of the state variables in the state space model are decoupled (or weakly coupled). Moreover we assume that the process dynamics allows the process to be simulated over the time horizon by means of the Euler forward integration method with 50 steps, and that 30 iterations (6 searching directions) in the optimization algorithm result in a control vector which is sufficiently close to an optimal value.

For the optimization algorithm based on the Maximum Principle, this leads to a time-consumption in the order of

$$
t_{1}^{c} \sim 3.6 * 10^{4} * n^{1 \cdot 2} \zeta
$$


where

$\zeta$ is the time consumption of a floating point operation

$n$ is the number of state variables and we assume that

$n$ is $2.5 r$

The corresponding expression for the algorithm based on minimizing the performance index by the use of a gradient technique is

$$
t_{2}^{c} \sim\left(2160 * n^{2 \cdot 2}+11160 * n^{1 \cdot 2}+9000 * n^{0 \cdot 2}\right) \zeta
$$

Using a Motorola 68020 processor with a floating point co-processor 68881, $\zeta$ is about $10 \mu \mathrm{s}$. With $n=10$ and $n=50$ the time consumption is

$$
\begin{array}{llll}
n=10 & t_{1}^{c} \sim 5.6 \mathrm{~s} & t_{2}^{c} \sim 5.3 \mathrm{~s} \\
n=50 & t_{1}^{c} \sim 39 \mathrm{~s} & t_{2}^{c} \sim 157 \mathrm{~s}
\end{array}
$$

The time consumption may seem alarming. However by increasing the efficiency of the optimization algorithm and accepting a less stringent solution the number of iterations can be reduced. Moreover faster processors can reduce the computation time to an acceptable quantity, and parts of the algorithms are well suited for parallel processing.

\section{Predictive control of a simple chemical reactor}

In order to try out alternative algorithms for predictive control, a lot of the simulations on an 'example process' have been carried out. Although the model contains non-linearities, linearization and the application of a PI-controller results in a satisfactory behaviour of this simple process in most cases. Our intention is to show that the predictive control algorithm can compete with conventional controllers even when simple processes are considered. A more realistic process with hard constraints on control variables and more dominating non-linearities is studied in another article (Balchen et al. 1988).

If an irreversible chemical reaction of $A \stackrel{k}{\rightarrow} B$ takes place in a continuous, well stirred reactor and the concentration of a component $A$ and the temperature in the reactor are defined as state variables with the rate of energy supply as the control variable, energy and mass balances result in a non-linear state-space model (numerical values are chosen from Perlmutter 1972). The total mass in the reactor is assumed to be constant, and the feed rate, the concentration of component $A$ and the temperature in the feed are considered to be the disturbances. In all the simulation results in Figs. 2 to 8 , both the concentration deviation of component $A$ from $0.3 \mathrm{~mol} / 1$ and the rate of energy supply are quadratically weighted in the performance index. Moreover a time horizon $T=0.5 \mathrm{~min}$ is used in the optimization algorithm and the control variable is represented by three parameters. The control variable is updated every $0.1 \mathrm{~min}$, even though the computational requirements outlined in the previous section, indicates that it would be possible to update the control variables 5-10 times as often.

In Figs. 2 to 5 the reactor is exposed to a stepwise increase in the feedrate of $18 \%$ at $t=1.0 \mathrm{~min}$. This can be due to some unpredictable changes (a destroyed valve for instance) or a conscious (predictable) change in the operating conditions. 


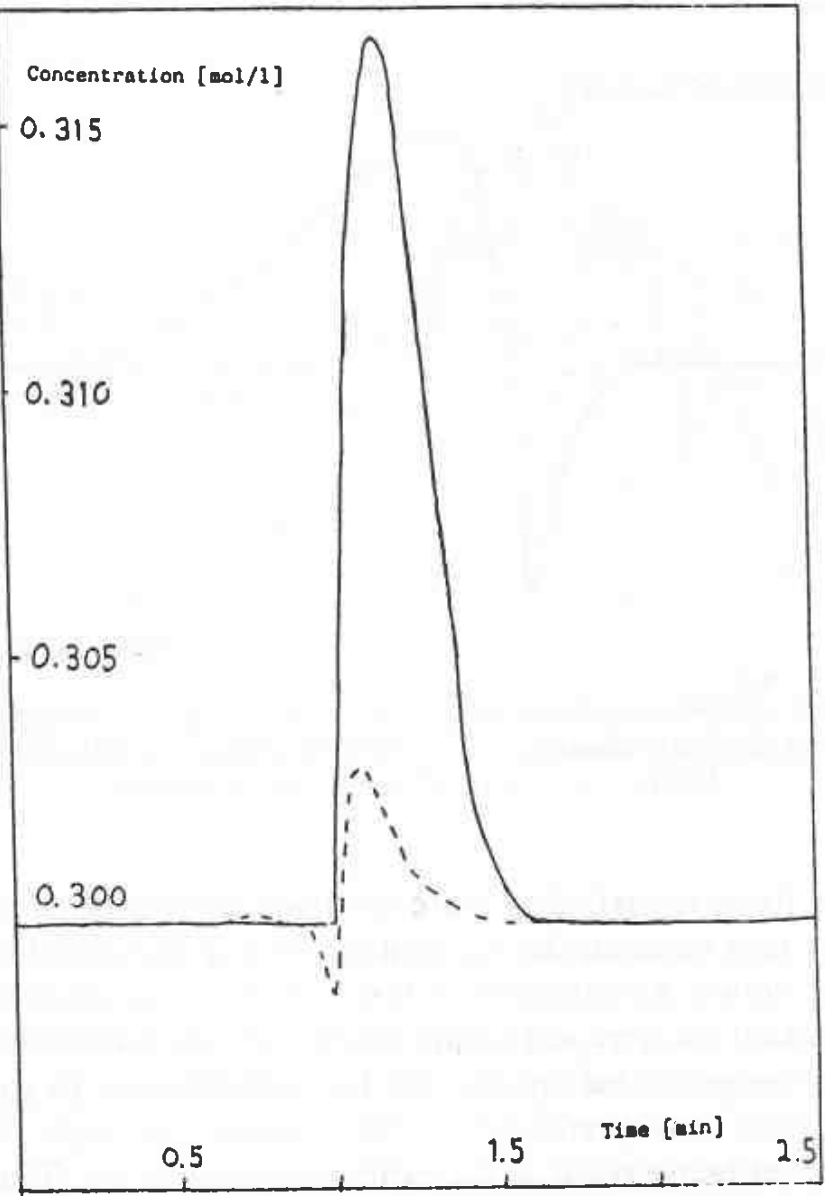

Figure 2. feedrate change not predicted, $G=(0) ;----$ feedrate change predicted, $G=(0)$.

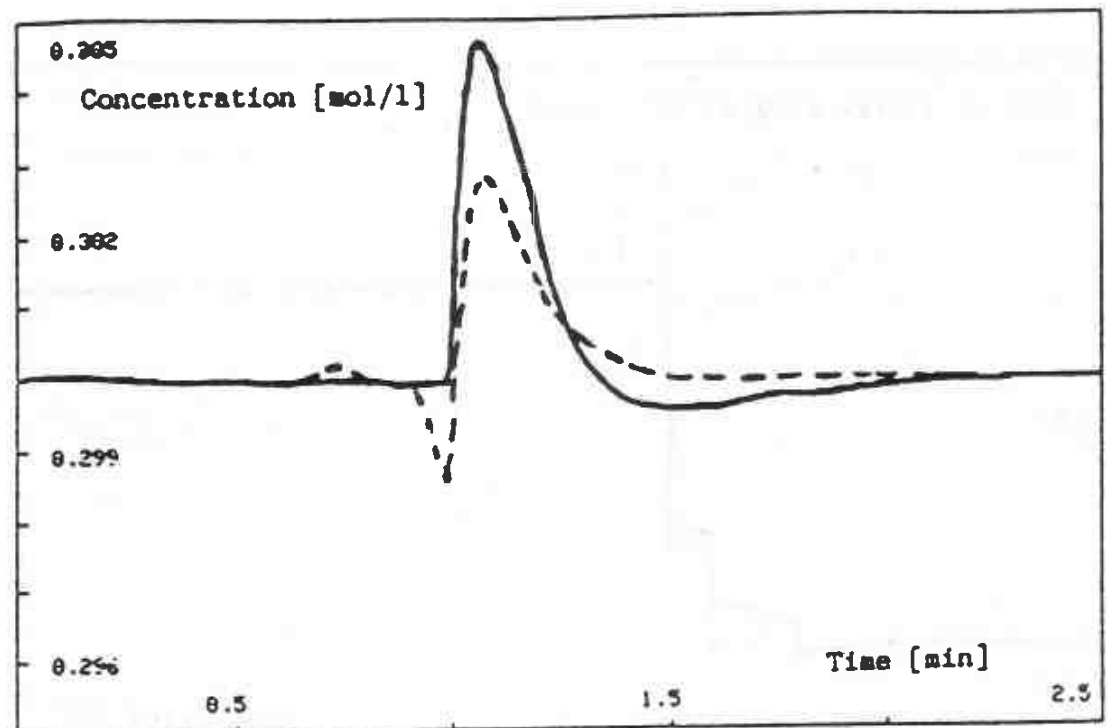

Figure 3. feedrate change not predicted, $g=286, g_{2}=-0.299 ;----$ feedrate change predicted $G=(0)$. 


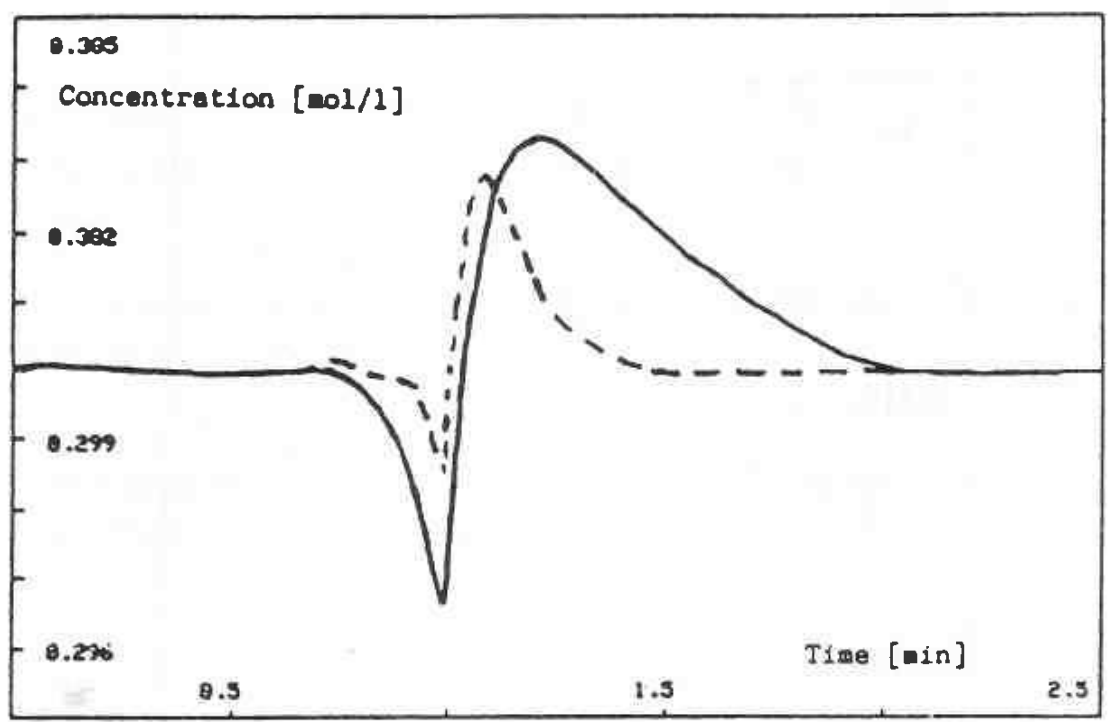

Figure 4. Predicted feedrate change $\longrightarrow$ rate of energy supply limited to $2.45 \times 10^{9}$ $\mathrm{J} / \mathrm{min} ;----$ rate of energy not bounded.

If the reactor form a part of a more complex industrial process the average feedrate may alter in a more stochastic manner. In Fig. 6 a possible deviation from the mean value is shown. An estimate of this deviation helps to control the reactor. We have assumed that we were not aware about how the disturbances have altered, and the estimate was generated by filtering the disturbances. In each optimization the disturbance then was predicted to be constant through the optimization horizon, the constant being given as the estimated value at the particular time. The simulation results are shown in Figs. 7 and 8.

Figures 2 and 3 indicate that a feedback matrix $G$ (ref. eqn. 6 ) is not necessary when it is possible to predict the disturbance, while the improvement is considerable when the stepwise change cannot be predicted.

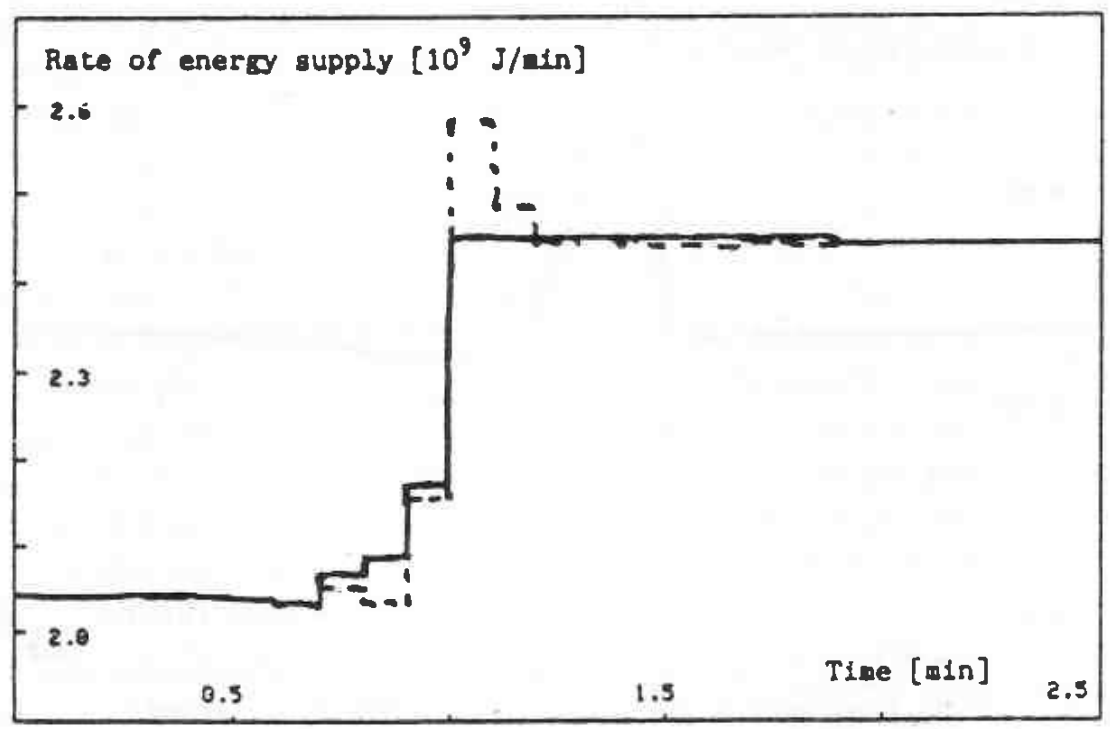

Figure 5. Rate of energy supply corresponding to the curves in Fig. 4. 


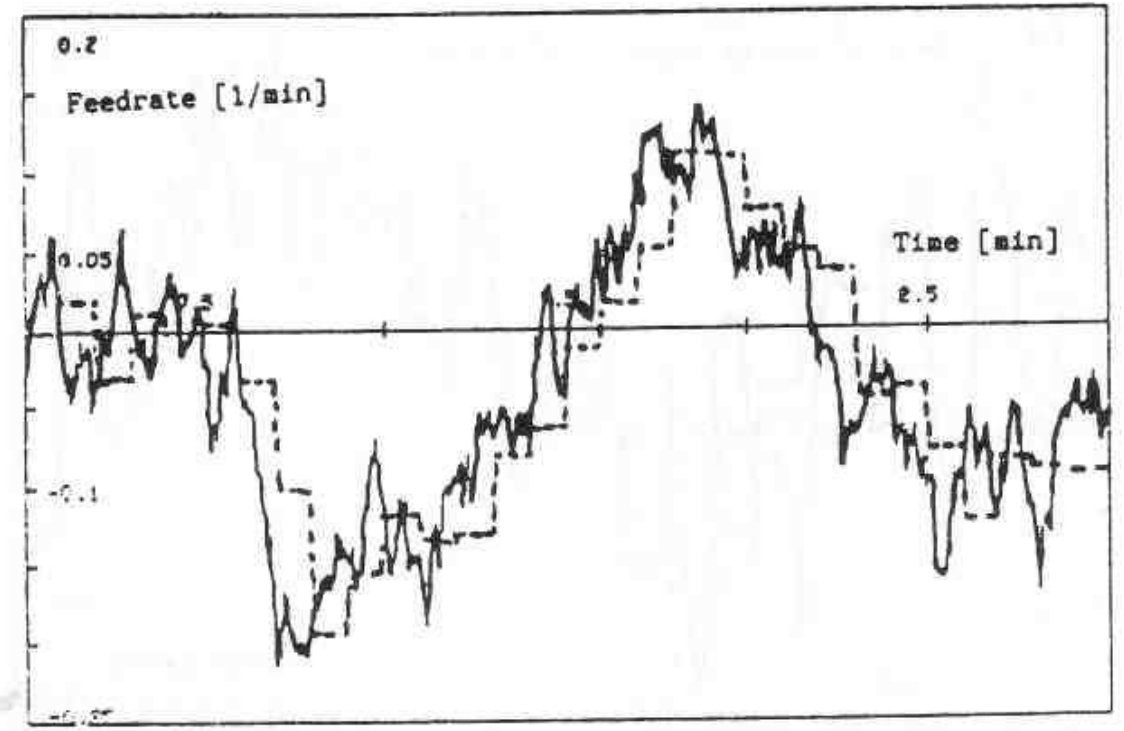

Figure 6. stochastic noise on the feedrate; - - - - estimated noise.

When the disturbance can be predicted and boundaries on the control vector are take into consideration, the process 'prepares' the stepwise change in the feedrate. This is shown in Figs. 4 and 5.

It is clear from Figs. 7 and 8 that instantaneous feedback through the $G$ matrix is of great importance when the process is exposed to rapid time-varying disturbances.

In Figs. 9 and 10 the concentration and rate of energy supply, resulting from an unpredictable stepwise change in the feedrate, are compared to the results achieved with a PI-controller. Due to non-linearities and dynamic coupling with the temperature, oscillatory behaviour is inevitable when the process is governed by a PI-

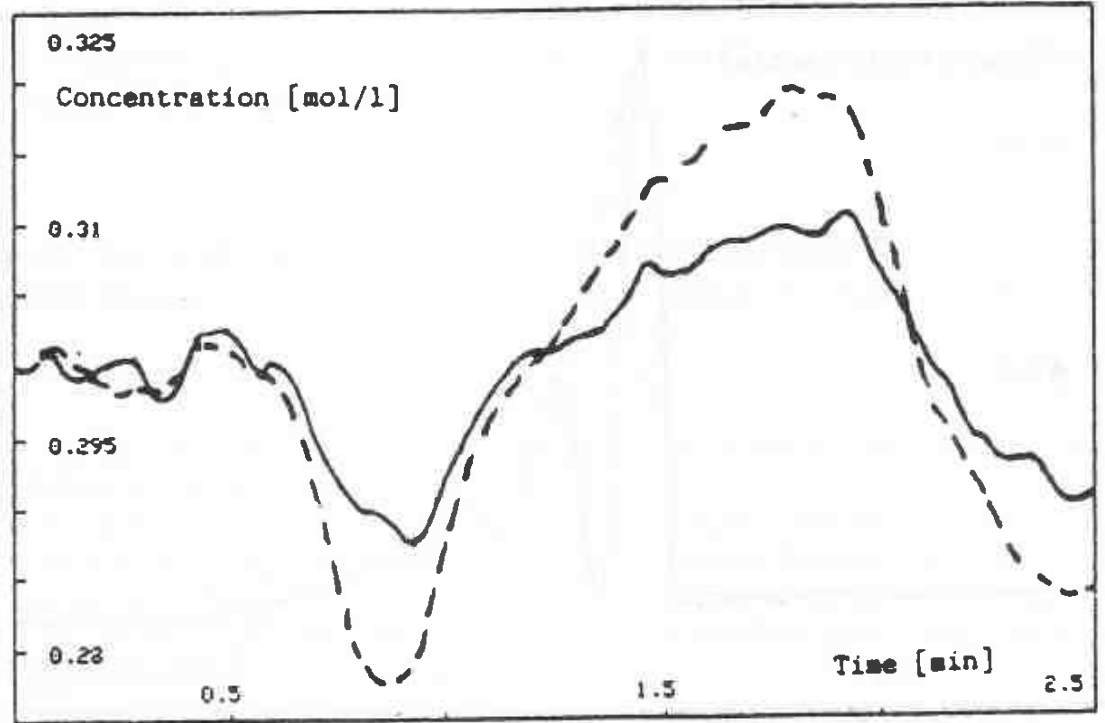

Figure 7. $-g_{1}=286, g_{2}=-0 \cdot 299 ;----G=(0)$. 


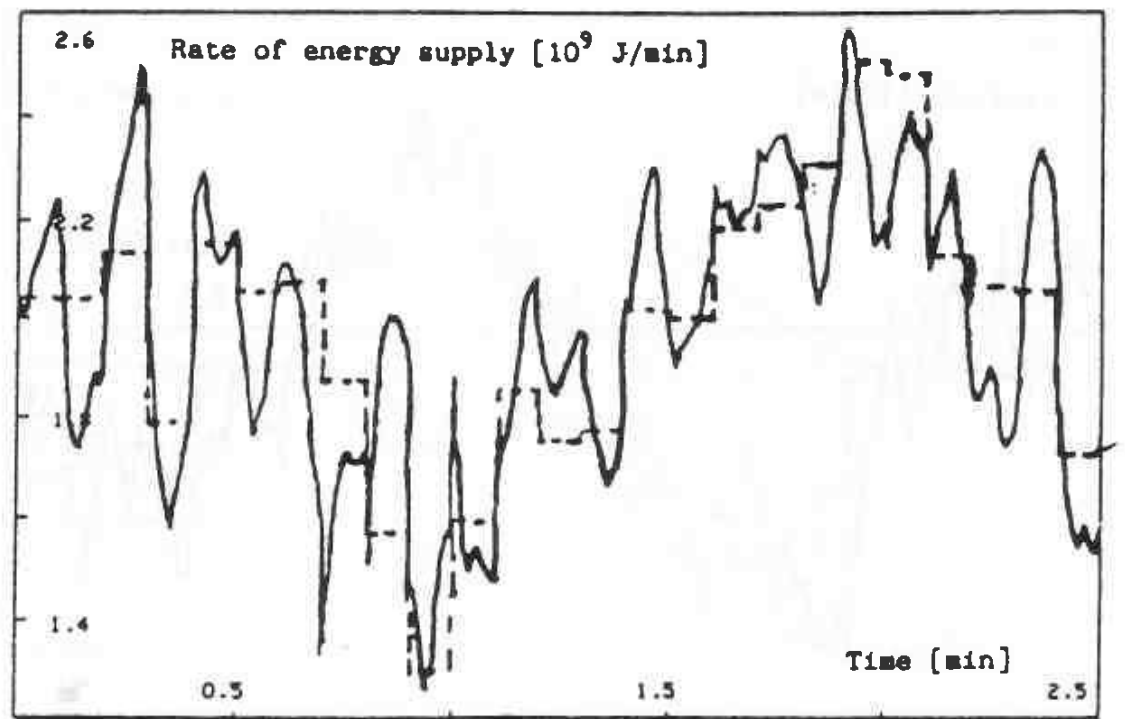

Figure 8. $g_{1}=286, g_{2}=-0 \cdot 299 ;----G=(0)$.

controller. It can be seen from Fig. 10 that predictive control results in a more economical means of controlling the energy supply. A PID-controller may be used to reduce the concentration 'amplitude'. However the same effect can be achieved with the predictive control algorithm by reducing the weighting factor for the rate of energy supply in the performance index. All in all we can conclude that the predictive control algorithm has shown results that are preferable to that of a PIDcontroller even for this simplified control problem.

\section{Conclusion}

Some principles for a predictive control strategy based on state-space models are presented in this paper. So far the effort has been directed at the optimization algorithm and parameterization of the control vector in order to keep the computation

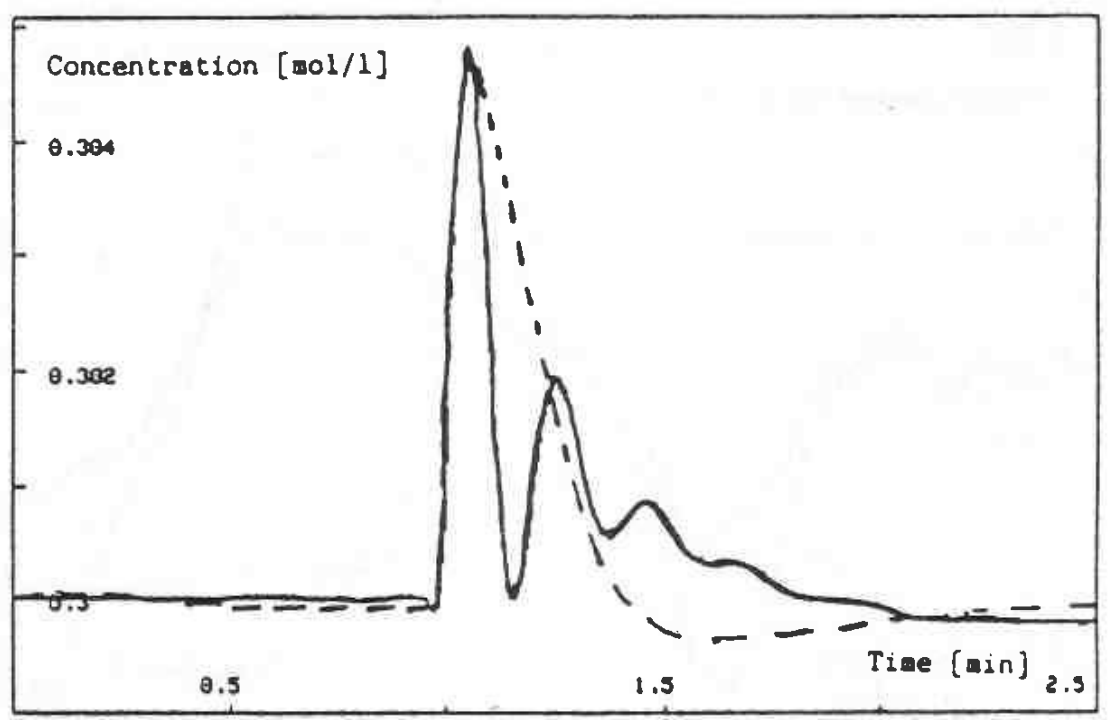

Figure 9. - PI control; - - - - predictive control, feedrate change not predicted. 


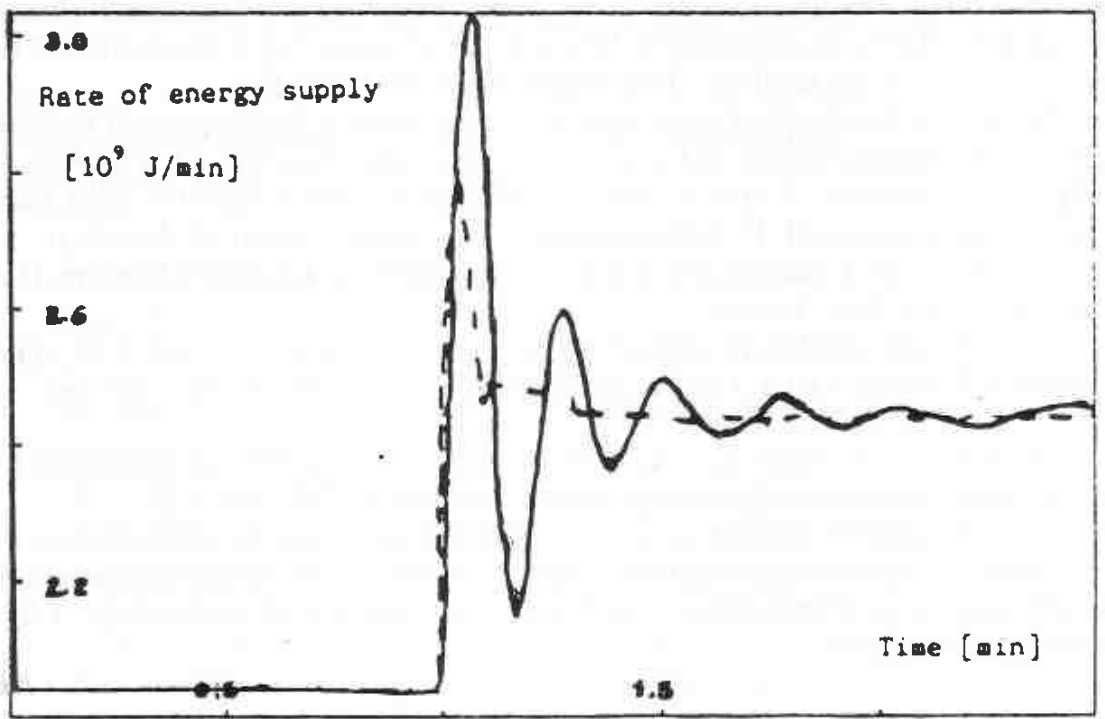

Figure 10. PI control; - - - - predictive control, feedrate change not predicted.

time to a minimum, which is extremely important to make the controlled system to function satisfactory.

The main disadvantage of the state space formulation is that a model structure must be assumed. However methods for structure identification may be applied. Since effective methods for parameter estimation exist and the model used in the optimization does not need to be extremely accurate. Optimal estimation of the state vector ensures optimal feedback control and representative estimates which can be used as initial values in the optimization algorithm.

In a practical situation it may be impossible to predict the dominating disturbances accurately. A control strategy that involves feedback from the estimated state-space variables should therefore be used. The time delay caused by the timeconsuming optimization algorithm is thus not so critical, but it has to be small enough to consider the constraints on the control and state variables in time. Even with a Motorola 68020 processor supported by the 68881 co-processor, processes with slow dynamics and not too many state variables can be controlled by the predictive control algorithm.

\section{ACKNOWLEDGMENT}

This work has been sponsored by The Royal Norwegian Council for Scientific and Industrial Research through the Predictive Control research program.

\section{REFERENCES}

Balchen, J. G. and Mumme, K. I. (1987). Structures in Process Control Systems, (Van Nostrand Reinhold Company Inc., New York).

BalChen, J. G., LJungQuist, D. and STRAND, S. (1988). State space model predictive control of a multistage electrometallurgical process, Modeling, Identification and Control, 10, $35-52$.

Clarke, D. W., Mohtadi, C. and Tufrs, P. S. (1987). Generalized predictive control-Parts I and II, Automatica, 23, 137-160.

CutLeR, C. R. and RAMAKER, B. L. (1980). Dynaic matrix control-a computer control algorithm, Joint Auto. Contr. Conf. Proc., paper WP5-B, San-Francisco, CA. 
Ljungquist, D. (1986). Predictive control based on Pontryagin's Maximum Principle (in Norwegian), Thesis (sivilingeniør) Division of Engineering Cybernetics, The Norwegian Institute of Technology, Trondheim, Norway, Des. 1986.

Marchetti, L., Mellichamp, D. A. and Seborg, D. E. (1983). Predictive control based on discrete convolution models, Ind. Eng. Chem. Proc. Des. Develop., 22, 488-495.

Mehra, R. K. and Mahmood, S. (1985). Model algorithmic control, chapter 15 in Distillation Dynamics and Control, ed. P. B. Deshpande (Instruments Society of America).

Perlmutter, D. D. (1972). Example 2.3 in Stability of Chemical Reactors (Prentice-Hall, Inc.; Englewood Cliffs, New Jersey).

REID, J. G. (1981) Output predictive algorithmic control: precision tracking with application to terrain following. AIAA Journal of Guidance and Control, 4, 502-509, No. 5, Sept.Oct.

Richalet, J. A., Rault, A., Testud, J. D. and Papon, J. (1978). Model predictive heuristic control: applications to industrial processes, Automatica, 14, 413-425.

STRAND, S. (1987). System for predictive control based of repetitive simulation on a dynamic model with a parameterized control vector (in Norwegian), Thesis (Sivilingeniør) Division of Engineering Cybernetics, The Norwegian Institute of Technology, Trondheim, Norway, March 1987. 